Original Research Paper

\title{
Evolution of Time Concept in Physics and in Philosophy
}

\author{
${ }^{1}$ Sivaram, C., ${ }^{2}$ Kenath Arun and ${ }^{2}$ Kiren, O.V. \\ ${ }^{1}$ Indian Institute of Astrophysics, Bangalore-560 034, India \\ ${ }^{2}$ Department of Physics, Christ Junior College, Bangalore-560 029, India
}

Article history

Received: 07-02-2015

Revised: 25-03-2015

Accepted: 25-03-2015

Corresponding Author:

Kenath Arun

Department of Physics, Christ Junior

College, Bangalore-560 029, India

Email: kenath.arun@cjc.christcollege.edu

\begin{abstract}
What is 'time'? This is a question that has perplexed many a physicist and philosophers alike. The perception of the passage of time appears ingrained in all biological systems where growth, evolution and decay of objects, etc. are measurable indicators of the passage of time. Here we look at various physical models of time, from Newtonian to relativistic. We also explore the idea of perception of time including the possibility of travel into the past and future. Time in the quantum world (as measured by differences in energy transitions of various levels) and classical world (as energy transformations) are also discussed.
\end{abstract}

Keywords: Newtonian Time, Relativistic Time, Steady State Universe, Multiverse, Tachyon, Closed Time-like Curves, Arrow of Time

\section{Introduction}

The perception of the passage of time, either periodic or continuous, appears ingrained in all living (biological) systems. The diurnal cycle and the annual passage of seasons create visible changes in ambient parameters influencing the behaviour of organized systems which perceive and respond to these changes. Growth, evolution and decay of objects, organisms or systems provide measurable indicators, of the passage of time. The advent and advance of humans, capable of meticulous observations of entities around them along with capacity for abstract thought enables quantitative measurement and recording of the passage of time.

Even early humans were aware of the regularity of the panoply of celestial objects discerning subtle motions of the moon and planets enabling at a later stage the accurate prediction of phenomena like eclipses. The Mayans deduced the orbital periodicity of Venus with an accuracy only improved in the twentieth century. Calendars to record events evolved early with meticulous book-keeping (in the temporal sense) of religious festivals, harvests, sowing of crops or even flooding of rivers. The year (associated with the earth's orbital period) was divided into twelve months (the lunar orbit) and months into weeks and days (for the diurnal earth rotation). Calendars as chronological indicators were crucial for culture, religion and all day to day activities. The need to measure time over the passage of a day led to the invention of clocks (Galileo's observation that the period of a pendulum depends only on its length speeded up development of accurate pendulum clocks improving on earlier devises like sundials, water clocks and hourglass sand clocks etc.).

The sexadecimal measure, introduced by the Babylonians applied both to measurements of angles and time, the hour being sixty minutes and a minute being sixty seconds. This is purely historical and any other number for subdividing would have been acceptable. Navigators at sea (during the great ocean voyages of the early European colonial power) needed to know their position on the globe and the passage of time more accurately. Measurements of latitude and longitude were begun. The definition of the metre in terms of the meridian passing through Paris led to more precise measurements. It was realized that local time was determined by longitude (as the earth rotates) and the Greenwich meridian was adopted as the longitude with respect to which times at all other places are measured (this of course led to GMT). Japan would be several hours ahead of GMT, while Hawaii would be several hours behind.

There is an amusing anecdote involving the International Date Line (IDL) which involves the addition and subtraction of a whole day depending on which direction one crosses it. This has to do with the global circumnavigation of the Magellan expedition (1519-1522). The captain Magellan was killed while battling the Pacific islanders, but much of the remainder of the crew now led by Juan Sebastián Elcano, managed to reach the starting point (Lisbon) of their voyage. Being strict Catholics, the crew were expected to keep 
track of all festivities like Lent, Easter, etc. and for this purpose had to maintain a log book. On their successful return (the first crew presumably to circumnavigate the earth) it was observed that they had missed one day, not accounted for. For this reason they were presumably pulled up by the orthodox clergy. Of course it was not their fault. They had no way of knowing that they had crossed the IDL and it was inevitable that they lose a day. Geographical time sense, based on longitudes had not yet reached the sophistication of the eighteenth or nineteenth centuries.

Local time based on geographical longitude further underwent changes. Countries like the U.S. or Russia, which spanned many degrees of longitude had to introduce time zones (where clocks could show different times depending on whether you are in the western or eastern part of the country.). When commercial air travel became common, this led to so called jet lags requiring time to adjust to the local time. Supersonic travel (like the now defunct Concorde) introduced the possibility of going back in time (at lease geographical time). You could have lunch in Paris and arrive for breakfast in N.Y on the same day. This has more to do with chronometric book keeping rather than actually becoming younger. Later on we will see that to really go back into the past one has to travel faster than light (superluminal). With advances in atomic and nuclear physics even clocks to measure small intervals (like a second) became very precise.

Incidentally one wonders how inhabitants of a planet like Venus (a day being longer than a year, a day of 243 earth days) would measure local time. On Mars it would be more reasonable (with a day near to $24 \mathrm{~h}$ ). Of course with the precision of atomic clocks, which can measure slow-down of the Earth's rotation, these questions are now academic, even for future colonisers.

\section{How Long is a Second?}

The advent of atomic clocks and the more recent nuclear thorium clock has enabled a second to be defined to an accuracy of one part in $10^{16}$ or $10^{17}$. The caesium clock has already been in existence for quite some time, the second being defined as 9,192,631,770 oscillations of the $\mathrm{Cs}_{137}$ hyperfine transition. This has enabled us to verify that the earth does indeed slow down by $1 / 6 \mathrm{~ms}$ per century, so that starting from 1972, leap seconds have been periodically added several times, the last in 2008 , so that new year 'celebrations' can last one second longer on December 31. This slow down is just what we expect from tidal action of the moon, whose distance consequently increases (from the earth) by about three centimetres a year. Thanks to laser ranging (accurate timing of laser pulses from reflectors on the moon) we know the distance to the moon to within a fraction of a centimetre. So also bouncing radar signals from the atmosphere of Venus has enabled the determination of the astronomical unit to within less than a kilometre. This accurate monitoring of time intervals has enabled unprecedented accuracy in measurement of times and distances. The millisecond pulsar, PSR B1937+21, (Backer et al., 1982) (rotating 642 times a second) is slowing down at the rate of one part in $10^{19}$, rivalling atomic clocks.

Atto-second laser pulses are now common, while yocto-second pulses $\left(10^{-24} \mathrm{~s}\right)$ have been monitored in connection with quark-gluon plasmas. Typical hadronic interactions are of $10^{-23} \mathrm{~s}$ duration. That gravity affects clocks was major offshoot of Einstein's General theory of Relativity (GR). A clock in a strong gravitational field runs slower, so a high altitude clock would run faster. You gain a pico-second over a lifetime by being in the topmost floor of a skyscraper.

\section{Concept of Time and Steady State Universe}

This brings us to the question of a time as a concept in physical theories. Early philosophers regarded time as flowing (into the future) like a river. Like in Tennyson's poem, men may come and men may go, but time goes on forever. Man was conscious of his mortality (his limited life on earth) and aspired to be immortal. So the concept of afterlife (after death.) was common to almost all cultures, from Voodoo Africans to Egyptians (who buried their kings in fantastically built pyramids with all paraphernalia) to the nirvana of Buddhists and multiple births and deaths in Hinduism till the soul attains immortality merging with the infinite. Even early cosmological ideas pictured the universe as without beginning or end. Hermann Hesse's novel 'Siddhartha' for instance is a well written account of the 'river analogy' flow of time. Fairy tales often ended with the key character being happy forever. Everlasting happiness, prosperity, etc. is what humans always aspired for.

In the steady state cosmology pioneered by British astronomers Bondi, Gold and Hoyle the universe has no beginning or end. They accounted for the Hubble expansion by postulating the perfect cosmological principle, which states that no matter where or when an observer studies the large scale universe, he would get the same unchanging picture. This implies a constant rate of expansion of the universe which in turn would imply a constant density at all times. The rate of expansion is given by Equation 1:

$H=\frac{\dot{R}}{R}=\left(\frac{8 \pi G \rho}{3}\right)^{1 / 2}$ 
For constant $H, \rho$ should be a constant. But since $\rho$ should decrease as the universe expands (taken to be an irrefutable observed fact.), matter should be continuously created in the space between the expanding galaxies. The rate of matter creation required can be easily estimated as (if $\dot{q}$ is the rate of matter created in a volume (as a function of time)) Equation 2 and 3:

$$
\frac{4}{3} \pi R(t)^{3} \dot{q}=4 \pi R(t)^{2} \frac{d R(t)}{d t} \rho, \rho \text { is a constant }
$$

Thus:

$$
\dot{q}=\frac{3 \dot{R}(t)}{R(t)} \rho=3 \rho H
$$

With the observed value of $\rho$ and $H$, this gives a vanishingly small value of $\dot{q}$ as $\sim 10^{-46} \mathrm{~g} / \mathrm{cc} / \mathrm{s}$, which is impossible to measure. The entire earth volume would increase in mass only a few $\mathrm{H}$ atoms per second. So in this picture the universe is timeless, without beginning or end. The older galaxies would drift further and further apart into oblivion and would be replaced by newer galaxies. We need not worry about beginning or end. Of course even in this steady state model, individual stars and galaxies continue to be born, evolve and die (ending up as black holes, white dwarfs or whatever.). Only the universe as a whole remains timeless. Galaxies may come and go, stars may come and go, but the universe goes on forever. Unfortunately for this pretty picture of the universe, the unambiguous observation of the Cosmic Microwave Background Radiation (CMBR) and its all-pervading presence and its isotropy, ruled out the steady state universe.

Although attempts were made by Hoyle and others to create a CMBR by iron or graphite grains thermalising star light (into microwaves), this contradicted observations. Especially the peaks and troughs of the CMBR observed by COBE and WMAP satellites were found fully consistent with the evolutionary model of the Universe (wherein it began in a hot dense phase and expanded as it cools, till the present radiation background temperature is a few degrees). However there may be still some comfort for a steady state picture, if as the latest observations seem to indicate, the universe could be ultimately dominated by a cosmological constant (a steady vacuum energy density with negative pressure, i.e., $P=-\rho c^{2}$ ), which would imply an indefinite steady state expansion at a constant rate in the distant future.

\section{Time and the Multiverse}

Again more recent theories of the earliest phases of the universe (which leads to a singularity in time and space at $t=0$, in the big bang picture) invoke a so called 'inflationary phase', dominated by a large vacuum energy (negative pressure) implying an early exponential expansion which diluted all unwanted relics (like monopoles.) apart from explaining the isotropy and 'flatness' of the universe which otherwise would imply 'unnatural initial conditions' (like equality of kinetic and potential energies to one part in $10^{30}$ or so at earliest times.). The universe pictured as a quantum vacuum fluctuation could arise spontaneously (at all times.). A total zero energy fluctuation would last arbitrarily long times (uncertainty principle.) and expand exponentially under negative pressure. The negative pressure energy (increasing with the expanding volume) would have to be compensated by creation of (positive energy) particles so that the total energy remains zero. So no violation of energy conservation. Hence the continuous creation in steady state theory (and sudden creation close to singularity at zero time in the Big Bang picture) is replaced by creation of matter in the exponentially expanding inflating phase.

The point is that these events of spontaneously forming universes could occur at all times anywhere. So depending on the initial conditions we could have at any instant, universes expanding or collapsing and evolving in all forms, perhaps with different coupling constants (of basic forces). They would all be causally disconnected from each other, so no signals could be exchanged among them.

The universes with the right conditions could support life, some of them leading to 'advanced' life with cognitive capacity to measure time. For instance a universe with a matter density ten times higher than ours could have collapsed long back while one with ten times lesser density would have expanded too fast to form large bound structures. This cosmic process of universes forming, evolving or disappearing would have always existed or would always exist to all eternity.

This also introduces a natural 'Darwinian' selection of universes, only some of which (perhaps an arbitrarily miniscule fraction) can sustain life. This is both philosophically and physically more attractive than to talk of a single universe ordained to start at some time in the past and now supporting life. This so called multiverse picture has gained currency and is also consistent with superstring models of the earliest phases of the universe where an arbitrarily large multitude of topologies are allowed with different physical evolutions in the future. Past, present and future have all become relative and subjective; the moment one brings in relativistic features (inertial, gravitational and cosmic) in the discussion. 


\section{Perception of Time Scales from Newton to Present}

Although now we talk of the universe being 13.8 billion years old (this being the age of precision cosmology, precisely with the CMBR temperature known to 5 decimals) it is remarkable to note that only well into the twentieth century did Western Astronomers conceive of or imagine time scales in billions of years. This was brought out after advances in nuclear physics (especially estimation of the ages of terrestrial rocks by radioactive decay of elements like uranium). Meanwhile nuclear astrophysics established that some stars including the sun are powered by thermonuclear reactions by conversion of Hydrogen to Helium (4 p $\rightarrow$ $\mathrm{He}^{4}$ ) allowing the sun to live another five billion years till it becomes a red giant.

Ironically even Isaac Newton thought that the world was created in 4004 BC (by adding up all time scales and ages of prophets in the Bible). Biblical time scales were widely prevalent and Bishop Berkeley even gave the date of creation as October 4, $4004 \mathrm{BC}$. When the sun's luminosity was estimated (and its mass calculated by Kepler's law) it became clear that even if it were fully made up of coal or fossil fuels it would burn out in five thousand years. Lord Kelvin and Wilhelm Helmholtz, two prominent physicists of 19th century, proposed that the sun generated its luminosity by gravitational contraction and estimated that if it had contracted from infinite dimension to its present size it would be about thirty million years old, which they claimed was reasonable.

This was already in conflict with Darwinian evolution theory (Darwin's book 'Origin of the species' appeared coincidently in 1859 around the same time, spurring a debate). The biologists needed a much longer time. It was only after radioactive determination of rock ages that the notion that the earth was about five billion years old was established.

Carl Sagan in his 'Cosmos' has pointed out that only the ancient Hindu culture conceived of time scales of billions of years. For instance, one Kalpa of Brahma is about 8 billion years, not far from the Hubble age. How they arrived at such long time intervals is not clear. Certain ancient Indian writings reveal on awestruck obsession with ideas of immense stretches of time.

A very curious example is given by $\mathrm{J} E$ Littlewood in his book "A Mathematician's miscellany", London, published more than sixty years ago. He quotes from Buckle's "History of Civilization in England" printed in the $18^{\text {th }}$ century (translating in English texts). It goes as follows: "There is a stone, a cubic mile in size, harder than diamond. Every million years, a holy man gives it the lightest possible touch. The stone is in the end completely worn away. The estimated time for this is $10^{35}$ years!.

Remarkably, $10^{35}$ years corresponds to the proton decay time in some of the more recent models. Earlier unified theories based on $\mathrm{SU}(5)$, for e.g., gave too low an age of $10^{31}$ years, subsequently ruled out by proton decay experiments. $10^{35}$ years is just beyond reach of experiments. The unification of quarks and leptons (the basic constituents of all matter) in these theories inevitably imply proton decay on such long time scales. So all composite particles like the proton must decay (all composite matter decay, the Buddha is supposed to have said). So nothing is forever. It is fantastic how such long time scales which only now (in recent years) are being recognized in modern physics were conceived of so long ago. So far proton decay is yet to be experimentally established unambiguously.

But are these the longest time scales imaginable? The evaporation times of large black holes due to Hawking radiation can be much longer. A solar mass black hole would evaporate completely on a time scale of $10^{71} \mathrm{sec}\left(10^{64}\right.$ years. $)$. The largest black hole mass conceivable is that comparable to the mass of universe. Even such a black hole should completely disappear by emission of Hawking radiation on a time scale of $\sim 10^{137}$ years. A galactic mass black hole would last for googol years, i.e., $\sim 10^{100}$ years.

Such a black hole would have a googolplex $\left(10^{10^{100}}\right)$ degrees of freedom describing its interior, generating the large entropy of black holes (through Boltzmann's formula). So these are conceivable Physical systems in which such large mathematical entities enter. The large entropy of black holes arises because of the drastic loss of information when matter collapses beyond the event horizon. When a solar mass collapses into such a state the entropy would increase by a factor of $10^{19}$. The baryon number is also lost. There is also a violation of time reversibility, i.e., such irreversible processes imply a time direction. Gravitational collapse into a black hole just implies such a time arrow (a future singularity). The expansion of the universe (from a past singularity) also gives a time arrow, a cosmological one. Even if such a universe collapses, the entropy would continue to increase (due to dissipative processes like collisions) and the future.

\section{Irreversibility and Arrows of Time}

It is of much interest that the first person perhaps to conceive of time as an extra dimension (on par with the three space dimensions) was not a physicist, but the well known writer, H G Wells. In his very first science fiction book, titled 'The Time machine' published in 1895, even in the introductory chapters he has the 
narrator (who actually built the time machine in his own yard.) explain to a group of acquaintances, how the machine is expected to work. The narrator suggests that 'time' should also be regarded as a dimension, in addition to the three usual co-ordinates (for instance, we can specify the location of a place in terms of latitude, longitude and altitude). Suppose a meeting is arranged at a particular venue, it is obvious the time should be mentioned in addition to the positional location. Hence four co-ordinates are required to describe the event and its evolution.

This was 10 years before, Einstein's relativistic electrodynamics paper of 1905 and 13 years before Minkowski's seminal work in 1908 on the fourdimensional space-time continuum and its relevance to the Lorentz transformation of special relativity, where rotations and boosts in this four-dimensional space-time describe all phenomena in special relativity. H G Wells who was also a science graduate of Imperial College London, also made the remarkable analogy (in his book) that the unidirectional flow of time (quite unlike the space co-ordinates) is similar to that of gravity acting only vertically downwards.

Even a stone falling is an irreversible process as Kinetic energy generates heat. So also collapse of stars heats them up, till nuclear reactions are triggered. Massive objects collapse to black holes, as beyond a particular mass the gravity mass cannot be balanced by any other forces. Gravity introduces time asymmetry and thermodynamic irreversibility. This leads to the thermodynamic arrow of time. All deterministic equations of motion in Newtonian mechanics (whether in Lagrangian or Hamiltonian form) are time reversible. One can always have a motion picture of the projectile or object run backward and it would look the same. But not when random motion is involved. If air resistance is considered, a projectile heats up, losing its energy to the random motion (heat) of air molecules (surrounding it). This leads to time asymmetry. We cannot put the heat (or random motion) back into the object. So also a falling egg gets smashed and cannot be put back.

This random motion or dissipation is not part of the least action principle. We have to postulate independently the second law of thermodynamics to supplement the description of the objects motion. This constant increase in unavailable energy characterizes entropy which causes more and more disorder. It is thus impossible to conserve time reversal invariance, if random motion dissipation is included. This also happens in Brownian motion (random walks), where the distance propagated by the particle steadily increases as the square root of time. So $t \rightarrow-t$, does not make sense. Similar thing happens in diffusion or heat conduction in a rod, where the system is described by a differential equation which is first order in $t$. Thus Equation 4: $\frac{\partial \varphi}{\partial t}=k \nabla^{2} \varphi$, or a diffusion process as

$\frac{\partial C}{\partial t}=D \nabla^{2} C$

( $C$ is the concentration $C(x, y, z, t))$.

$t \rightarrow-t$ does not preserve the symmetry. There is a time arrow inherent in the phenomena (we cannot put back the heat in the brick or rod). On the contrary, the differential wave equation, describing the propagation of a wave $W$ (with velocity v) is second order in $t$ Equation 5:

$\frac{\partial^{2} W}{\partial t^{2}}=\frac{1}{\mathrm{v}^{2}} \nabla^{2} W$

So that is invariant under $t \rightarrow-t$.

Same thing holds for periodic motion of a pendulum, with kinetic energy and elastic energy being interchanged ad infinitum. However a damping force, say proportional to velocity, introduces a first order in $\mathrm{t}$, so the motion is not periodic and is not time symmetric. Similar thing happens to a damped wave. The phenomena extend to electric circuits (RLC circuits), electronic devices, hydraulic systems, biological processes etc. As we stated above, recollapse of the universe is not time- symmetric as considerably more entropy is generated and as Penrose has pointed out, if one considers all the black holes formed, the chances of such a collapses leading to the re-emergence of an identical universe, as before the collapse, is only 1 part in $10^{10^{123}}$.

It is like mixing a spoon of oil into the ocean (at a given instant) and hoping to recover it (impossibly low probability). In Poincare's ergodicity hypothesis, given enough time, all parts of phase space could be sampled, in an arbitrarily large recurrence time. In general interacting many-body systems inevitably imply a time arrow. The collapse of wave function is also associated with a time-arrow (the quantum arrow of time) as the initial state is unrecoverable.

We also have the biological arrow of time (organisms continue to age, even if in the same location of space, like a tree.). The chronological arrow (measured by clocks) is also asymmetric of time, telomere shortening is also irreversible. Loss of memory of events, etc represents conscious arrow of time.

\section{Newtonian and Relativistic Time}

Newton considered time and space as immutable. $\mathrm{He}$ wrote in Principia, "Absolute, time and mathematical Time, of itself and from its own nature, flows equably without relation to anything external". Einstein changed all this notion of absolute time that exists independently of everything else. He showed and 
he has the backing of a century of experimental support, that time is relative. The only way the vacuum speed of light is the same for every observer in uniform motion, is if time passes at different rates for different observers. Now thanks to the GPS system, these effects are manifested in everyday life. If you take an intercontinental transatlantic flight, when you get off the plane you would be ten nanoseconds younger than those that saw you off at the airport.

An astronaut who visits the International space station for two weeks would return 500 microseconds younger relative to people on ground. Both special and general relativity come into play, when GPS receivers estimate locations accurately. Ignoring these effects (of gravity or velocity on time) can cause considerable errors. The most dramatic effects of relativity on time occur at speeds very close to that of light. A round trip to the star Vega at 0.99 light speed would enable the astronaut to gain 45 years, compared to people who stayed on, at the earth.

Subatomic particles like muons, produced in cosmic rays striking the Earth's upper atmosphere, travel close to light speed and decay at much slower rates (than when at rest) enabling them to survive to the Earth's surface. An observer commoving with the particle would see the muon cover a much shorter distance. These effects evidently manifest themselves in accelerators like SLAC, where there was no problem in aligning the electron beam (to travel kilometres) at $40 \mathrm{GeV}$, as in the frame of the electron, distance moved is hardly a meter.

A neutron with energy $\sim 10^{19} \mathrm{eV}$, would have a gamma factor $\gamma$ (Lorentz factor) of about $10^{10}$, so its lifetime would be dilated to $\sim 10^{13}$ seconds and can thus cross the galaxy. Its proper lifetime is still only around thousand seconds. The interpretation is "In the proper (commoving) frame of the neutron, the distance travelled is only $10^{23} \mathrm{~cm} / 10^{10} \approx$ Diameter of galaxy/10 $0^{10} \approx 10^{13}$ $\mathrm{cm}$, i.e., proper lifetime $\mathrm{x}$ velocity of light $\approx 10^{13} \mathrm{~cm}$ ".

However the outside observer sees it moving a distance $3 \times 10^{23} \mathrm{~cm}$, i.e., he sees its lifetime dilated to $10^{13} \mathrm{~s}$, so it travels a distance, $3 \times 10^{23} \mathrm{~cm}$, travelling the galaxy. The neutron has moved only $10^{13} \mathrm{~cm}$, as measured in its frame. So distance contraction and time dilation are equivalent. Going arbitrarily close to the speed of light, it is possible for an astronaut to travel to the Andromeda galaxy in thirty years (of proper time).

The $\gamma$ factor would be $\sim 10^{5}$, so a one ton spaceship would have to expend $10^{26}$ Joules of energy, equivalent to mankind's power consumption (at present rate) for a few million years. To travel to Alpha Centauri and back within 20 years, the energy to be supplied per unit mass of payload is a billion times that of Apollo flight to the moon. Expending such vast amounts of energy (even for very modest payloads) is well beyond contemporary technology. Besides the power and infrastructure required for a one ton space probe to reach Alpha Centauri in fifty years, the cost of the trip would be several hundred trillion dollars. It involves several thousand tons of high energy fuel.

So literally time is money for such relativistic trips. If you want to cover vast interstellar distances in your lifetime, the costs would be super-astronomical and it does not increase linearly. This is also true in more mundane situations. Supersonic flight would be much more expensive than say train travel. Walking would be the cheapest. So what one gains in time, one loses in money spent. Time is money. Even for interplanetary space crafts, increasing the energy supplied, say for a Martian trip, by four times, can reduce the time travel by one-fourth (the spacecraft would take seventy days to reach Mars, rather than two hundred and fifty days for the lowest energy trips).

Photons and particles travelling exactly at light speeds do not 'sense' time. Einstein pointed this out in one of his first Gedanken experiments, what happens to an observer who moves away from a clock at exactly light speed. He would see the distant clock, always showing the same time. So no time elapses in the frame of an observer moving with photon. Photons are timeless so also gravitons.

Gravity can also slow down light. This has been measured by the time elapsed by signals from distant spacecraft (like Cassini) as they pass the gravitational field of sun, when reaching earth. As one approaches the event horizon of a black hole time slows down and vanishes at the horizon.

On the surface of a neutron star, time runs at a rate about thirty per cent slower than it does on the earth. If a spaceship is piloted close to a black hole event horizon, you can slow down time to any rate you want, depending on how close you come to the horizon. A distant observer sees all events halt at the horizon; time actually stops there. This is analogous to the velocity equalling light velocity in special relativity. A distant observer sees an observer falling freely into the black hole, take infinite time to reach the horizon, whereas the falling observer can cross the horizon and hit the singularity in a finite time.

A time traveller would only want to get near the event horizon, not cross it. As time slows down to almost a stop, very near the horizon, astronauts could see the universe's entire history pass before their eyes. Astronauts could in the future be able to travel superfast by diving close to a black hole and age much more slowly, returning to earth in the distant future. This is only a theoretical discussion as the tidal forces in the vicinity of the black hole will be extremely large to break-up the spacecraft, making this scenario practically impossible. It should also be noted that the 
decelerations required would also be too great for a human being to withstand.

So also astronauts in relativistic spacecraft approaching arbitrarily close to light velocity could survive and return to earth, some billion years in the future. The earth itself might have ceased to exist when they return. Travel into the very distant future is certainly feasible in special relativity (and GR with gravity assists of black holes, etc.), but prohibitively expensive. The astronaut can literally travel the entire universe in his lifetime and return to see no vestiges left of the parent planet he took off from.

\section{Time Travel to the Past: Faster than Light Speeds?}

What about travel into the past? For this one requires velocities faster than light. One can visualize this, simply as follows. An observer moving with a beam of light (at light velocity) always sees the clock fixed at the same time. Suppose an observer could be transported to Andromeda, in hundred years, at one million times light velocity. When he looks at the earth, he would see it as it was in the distant past, hundred million years ago and perhaps could see dinosaurs roaming about. So he has moved into the distant past by travelling much faster than light. Indeed it is easy to show that a tachyonic message would be received by an observer in reverse order. That time travel into the distant past is indeed possible with faster than light (tachyonic) velocities is easily seen as follows (Feinberg, 1970).

The Lorentz transformation of space $(\Delta x)$ and time $(\Delta t)$ intervals in some Inertial Frame (IF), corresponding to primed quantities $\left(\Delta x^{\prime}, \Delta t^{\prime}\right)$ in another frame moving with velocity $\mathrm{v}$ along $\mathrm{x}$-axis is given by Equation 6 and 7:

$$
\begin{aligned}
& \Delta t^{\prime}=\gamma\left(\Delta t-\frac{\mathrm{v}^{2}}{c^{2}} \Delta x\right) \\
& \Delta x^{\prime}=\gamma(\Delta x-\mathrm{v} \Delta t)
\end{aligned}
$$

Where:

$$
\gamma=\frac{1}{\sqrt{1-\mathrm{v}^{2} / c^{2}}}
$$

If the velocity ' $u$ ' of an object moving in the original frame is $\Delta x / \Delta t$, we get the frame velocity $v \leq c$, but $\Delta t$ ' can have a sign opposite to $\Delta t$, if $u / c>c / \mathrm{v}>1$.

We can picture this result as a continuation of the usual time-dilatation picture. The slower time flows, the faster one travels from rest; time stands still for travel at velocity of light and time runs backwards once $\mathrm{C}$ is exceeded. When a tachyon, with $u>c$ is emitted by a source moving backwards with speed $v>c$ (and following $u / c>c / v>1)$, the tachyon would reach its target a time $\Delta t^{\prime}$, before it was sent out.

Then if returned by a similarly backward moving source, it would arrive at its starting point, $2 \Delta t^{\prime}$, before emission. So such tachyonic time machines, to travel to the past are physically consistent provided tachyons exist. No tachyons have yet been experimentally detected. However Einstein's GR, also has solutions which involve closed time loops, or closed time - like curves, where one can return to the starting point in time just as if one moves along a closed curve or path, one can return to the starting location.

\section{Closed Time-Like Curves and Time Travel to the Past}

The Gödel universe (Gödel, 1949) is one such solution with closed time-like curves which may permit construction of time machines. So there can be geometries containing paths along which one can travel into the past with velocity less than that of light. Note that unlike in SR, where Faster Than Light (FTL) velocities are required to travel to the past, in General Relativity (GR) one can go into the past with velocities less than light velocities. It is not surprising that Einstein's GR allows Closed Time-like Curves (CTC), because in GR geometry is determined from matter. (Although the "Chronological Censorship" implies that nature does not admit and cannot admit time loops (Bronnikov and Rubin, 2012))

So we can always choose an interesting geometry even if very peculiar, containing CTC's. We can then construct the matter distribution (very unphysical.) which gives rise to such geometries. So a time machine solution, like Gödel universe, or Einstein-Rosen bridges or wormholes always entails exotic matter distribution. For instance, Gödel universe requires constant and uniform energy distribution, which is unphysical. Wormholes, which are threaded by closed time like curves, connect distant regions of Space-Time, but required matter with arbitrarily large negative energy densities, to avoid their mouths closing cutting off all paths through them (Morris and Thorne, 1988).

It has also been hypothesized that space-times around cosmic strings could generate Closed Time like Curves (CTC's) (Vilenkin, 1985). The area is vanishingly small (thin structure) with a mass density proportional to a $2 \mathrm{D}$ spatial $\delta$-function and could be endowed with intrinsic spin $j$ per unit length. The solution is a 2-parameter $(m, j)$ metric tensor and as in the case of the Gödel line element there is no structure in the $\mathrm{z}$-direction, while in the perpendicular $(r, \theta)$ plane, with $\theta=0$ and $2 \pi$ defined, the 
non-trivial geometry supports CTC's when $J \neq 0$. By changing co-coordinates the space-time can be made locally Minkowskian, with Equation 8:

$$
t^{\prime} \rightarrow t+\frac{4 G}{c^{4}} j \theta
$$

With defect angle Equation 9:

$$
\varphi=\left(1-\frac{4 G m}{c^{2}}\right) \theta
$$

The new time variable $t$ ' rather than flowing smoothly in a linear fashion jumps by $8 \pi G j / c^{4}$ when the string is circumnavigating. This is similar to the picture of time at the quantum level as a torsion induced defect in space-time (de Sabbata and Sivaram, 1994; 1996; Sivaram et al., 2014), time jumping in steps of the Planck time Equation 10:

$$
t_{P l}=\left(\frac{\hbar G}{c^{5}}\right)^{1 / 2}
$$

I.e., we had the relation $\int_{S} Q d A$, over a surface $\mathrm{S}$, $Q=\frac{4 \pi G}{c^{4}} \hbar, \hbar$ being the intrinsic unit of spin, $Q$ being the space-time torsion.

In particular (also in crystals.) torsion causes dislocations in space, whereas curvature causes disinclinations. If one circumnavigates a closed surface with curvature $\kappa$, over an area element $d A$, there is a change in direction by parallel transport of $\int \kappa d A=\theta$, for a flat surface the vector still points in the same direction.

In addition if space-time has a torsion, the vector not only changes direction but is displaced by $\delta l$ to a different position, $\int Q d A=\delta l$ and this can be in quantum units. The time defect is defined as $\frac{1}{c} \int Q d A \approx n t_{P l}$, in multiples of Planck time. This suggests a possible basis for the directionality of time.

The situation could be analogous to a ferromagnet, where when the temperature drops below $T_{C}$, all spins are aligned, giving a residual (net) magnetism. Perhaps in the very early universe, close to the Planck epoch, the time dimension, which was earlier pointing in all directions like the spatial coordinate, got 'locked' (froze) in one direction, when the energy (temperature) in the early expanding universe dropped below a threshold. A space-time phase transition occurred, especially if time was pictured as arising due to dislocation defects and a propagating torsion field $(Q=2 \mu \varphi)$ had a similar structure to the Higgs field with a quartic structure and the rotational symmetry was spontaneously broken for the time co-ordinate.

This symmetry breaking would have released 'latent vacuum energy of the ground state', causing an exponential expansion in the time-coordinate thus fixing the time arrow. The details are being explored and would be reported elsewhere (in preparation 2014).

\section{Quantum Time Limit}

The existence of a lower quantum limit for time, i.e., $t \approx t_{P l}=10^{-43} \mathrm{~s}$, also has many implications, like an upper limiting temperature $T_{P l}=10^{32} \mathrm{~K}$, upper limits to field strengths and consequences for the singularity in the early universe (Sivaram and Arun, 2011; 2012). Again the temperature-time uncertainty relation has been explored in de Sabbata and Sivaram (1992a; 1992b). This has implications in quantum field theory where time is the inverse of the thermal Green's function.

The quantum nature of time would also have implications for CTC's. Even if one tries to return to the past event in a CTC, there would be an intrinsic quantum uncertainty, so that one misses the particular event (like killing one's grandparents.) The other possibility is that antiparticles (e.g., positrons) travel backwards in time, a la Feynman, so by going back in time, you could be antimatter and annihilate the target. Hawking has also argued that quantum fluctuations around a wormhole generate infinite stress thereby closing it. Moreover the narrowness of the channel would prevent anything macroscopic from squeezing through the opening. Moreover, there is Hawkins's Chronology Protection Conjecture (CPC) to prevent CTC's (Hawking, 1992).

This applies to a wide range of space-times. Particles or fields falling to a Kerr black hole or travelling around spatially closed Taub-NUT or Misner space become initially blue shifted as they near the horizon and resultant divergent energy density will always act back on the space-time to prevent CTC's from forming.

There have been recent proposals to circumvent this such as Alcubierre's space warp, Krasnikov tubes, etc. A space warp, hundred meters wide and ten meters thickness, requires more than $\sim 10^{45}$ Joules to keep its ends open. This is more radiation than what the Sun would emit in its lifetime. So it looks as if travel into the past requires colossal energies, similar to relativistic travel into the future. Suspended animation could be a biological way of time 'dilatation', deep freezing an individual (like cyborgs.) and reviving after several centuries or millennia (depending on the progress in medicine). It is significant that DNA of primeval organisms have been preserved (in amber) in fossils 
dating several hundred million years. The organism (including perhaps humans) could be cloned after arbitrarily long period in spacecrafts.

\section{Cosmic Time}

A notable feature of homogenous and isotropic expanding space-time like the Robertson-Walker metric which describes our present universe is the presence of a universal cosmic time for all observers (at all locations) at a given epoch. This arises because of the separability of space and time in Gaussian coordinates. This leads to the cosmological principle wherein at a given epoch, the universe should appear the same to all observers irrespective of their location (the two dimensional analogy is an expanding balloon painted with dots, each epoch being characterized by the scale factor, roughly the balloon size as it expands). So at $t_{1}$, we have the surface $R\left(t_{1}\right)$, all observers on 2-d surface have the same cosmic time $t_{1}, R\left(t_{2}\right)$ the surface at time $t_{2}$ in the expanding space, again same for all observers. So the 'history' of universe is a succession of two surfaces evolving in time.

For the 3-d case, which is our universe, the history is a succession of 3-d hyper surfaces evolving in time, expanding to larger and larger volumes $2 \pi^{2} R^{3}(t)$. The homogeneity and isotropy is confirmed by observations especially concerning the CMBR. There is no need for cosmic models, with different rates of expansion (anisotropic) in different directions. It is thus remarkable that though we have a plethora of time zones (for geographic convenience) on earth, there is a universal cosmic time (for all observers) characterizing each instant of expansion of universe. Thus all observers wherever they are located at the present epoch (Andromeda, M87 etc) would agree that the universe has been expanding for 13.7 billion years. We also live in a flat universe (spatial curvature vanishing, but not on a flat earth.

\section{CP And T Violation}

It was just 50 years that the CP symmetry violation was discovered in particle physics in the decay of long lived neutral $\mathrm{k}$ mesons (Christenson et al., 1964). From the CPT theorem, this is tantamount to violation of time reversal (T) symmetry. One of the unsolved puzzles of cosmology is the drastic asymmetry between baryons and anti-baryons in the present universe. If there were equal amounts, i.e., zero baryon number (in all high energy processes equal numbers are produced). The universe would be all radiation and life would not exist. So initially there was a small violation in $\mathrm{B}$ conservation, i.e., the number of baryons exceeded anti-baryon number by one part in 109, (say). It was pointed out by Sakharov that not just $\mathrm{B}$ number violation is required, but also $\mathrm{CP}$ violation apart from a non-equilibrium dynamics. We saw that in the collapse of matter beyond the horizon of a black hole, baryon number is violated (all baryons disappear in short time.) as well as $\mathrm{T}$ violation which is equivalent to $\mathrm{CP}$ violation.

It is possible that evaporating mini black holes in the early universe could have provided a baryon initial asymmetry (Sivaram, 1982). Again presence of an electric dipole moment for an electron or neutron could indicate $\mathrm{T}$ violation as argued by Landau and others.

\section{Conclusion}

In brief, the entity loosely called ' $T I M E$ ' continues to be profound and mysterious in its concept, passage and measurement on all scales of the universe, classical and quantum. Time in the quantum world is measured by differences in energy transitions of various levels, $\Delta E$, of atomic, nuclear or particle interactions. Classically, energy transformations or flows of heat fluxes or wave energies measure time. A uniform universal energy state, unevolving and not exchanging or transforming energy is dead in time.

\section{Acknowledgment}

The authors express their thanks to Dr. Ioannis Haranas and Dr. Ioannis Gkigkitzis for inviting us to contribute to this special issue and to the referee for the constructive feedback.

\section{Author's Contributions}

All authors equally contributed in this work.

\section{Ethics}

This article is original and contains unpublished material. The corresponding author confirms that all of the other authors have read and approved the manuscript and no ethical issues involved.

\section{References}

Backer, D.C., S.R. Kulkarni, C. Heiles, M.M. Davis and W.M. Goss, 1982. A millisecond pulsar. Nature, 300: 315-318. DOI: 10.1038/300615a0

Bronnikov, K.A. and S.G. Rubin, 2012. Black Holes, Cosmology and Extra Dimensions. 1st Edn., World Scientific, Singapore, ISBN-10: 9814374202 , pp: 427.

Christenson, J.H., J.W. Cronin, V.L. Fitch and R. Turlay, 1964. Evidence for the $2 \pi$ Decay of the $K_{2}^{0}$ Meson. Phys. Rev. Lett., 13: 138-140. DOI: 10.1103/PhysRevLett.13.138 
de Sabbata, V. and C. Sivaram, 1992a. Curvature from spin. Foundat. Phys. Lett., 5: 579-584. DOI: 10.1007/BF00665938

de Sabbata, V. and C. Sivaram, 1992b. Anyons and torsion. Nuovo Cimento B, 107: 1201-1205. DOI: 10.1007/BF02727205

de Sabbata, V. and C. Sivaram, 1994. Gravity and CP violation. Foundat. Phys. Lett., 7: 475-482. DOI: $10.1007 / \mathrm{BF} 02189250$

de Sabbata, V. and C. Sivaram, 1996. Twistors, torsion and quantization of space-time. Nuovo Cimento A, 109: 377-390. DOI: 10.1007/BF02731087

Feinberg, G., 1970. Particles that go faster than light. Scientific Am., 222: 69-76. DOI: $10.1038 /$ scientificamerican0270-68

Gödel, K., 1949. An example of a new type of cosmological solutions of Einstein's field equations of gravitation. Rev. Modern Phys., 21: 447-450. DOI: 10.1103/RevModPhys.21.447

Hawking, S.W., 1992. Chronology protection conjecture. Phys. Rev. D, 46: 603-611.

DOI: 10.1103/PhysRevD.46.603
Morris, M.S. and K. S. Thorne, 1988. Wormholes in spacetime and their use for interstellar travel: A tool for teaching general relativity. Am. J. Phys., 56: 395-412. DOI: 10.1119/1.15620

Sivaram, C., K. Arun, O.V. Kiren and B.N. Sreenath, 2014. Gravity of accelerations on quantum scales and its consequences. General Phys.

Sivaram, C. and K. Arun, 2011. Enigmatic aspects of the early universe: Possibility of a 'pre-big bang phase'. Astrophys. Space Sci., 333: 9-10.

DOI: $10.1007 / \mathrm{s} 10509-011-0670-5$

Sivaram, C. and K. Arun, 2012. Extended equivalence principle: Implications for gravity, geometry and thermodynamics. Hadronic J., 35: 653-659.

Sivaram, C., 1982. Asymmetric lepton production in a universe with non-zero baryon number. Astrophys. Space Sci., 82: 485-488. DOI: 10.1007/BF00651456

Vilenkin, A., 1985. Cosmic strings and domain walls. Phys. Rep., 121: 263-315.

DOI: $10.1016 / 0370-1573(85) 90033-X$ 